\title{
MOEMS Tuning Element for a Littrow External Cavity Laser
}

\author{
Richard. R. A. Syms, Senior Member, IEEE, and Anke Lohmann
}

\begin{abstract}
A miniature grating-tunable external cavity laser diode constructed using microoptoelectromechanical systems (MOEMS) technology is described. The tuning element is a vertically etched blazed grating mounted on a compound flexure, which consists of a cantilever in series with a portal frame. The flexure is deflected using comb electrostatic drives to rotate and translate the grating. The tuning element is prototyped using deep reactive ion etching of bonded silicon-on-insulator (SOI) material. Interferometric measurements of electromechanical performance are presented, and departures from the ideal behavior are identified. Electrostatic tuning of a Littrow external cavity laser over a range of $20 \mathrm{~nm}$ using a $50-\mathrm{V}$ drive is demonstrated.

[1000]
\end{abstract}

Index Terms-External cavity, grating, microelectromechanical systems (MEMS), microoptoelectromechanical systems (MOEMS), semiconductor laser.

\section{INTRODUCTION}

$\mathbf{T}$ UNABLE lasers are essential elements of wavelength multiplexed optical systems. However, the powers available from monolithic tunable sources such as multisection distributed Bragg reflector lasers [1] are often lower than fixed devices, and tuning may require a complex control algorithm based on stored calibration data [2]. External cavity lasers may have the advantage of higher output, a simpler tuning algorithm, reduced modal noise during tuning, and reduced sensitivity to aging.

Microoptoelectromechanical systems (MOEMS) technology allows electrical, mechanical and optical functions to be combined in a stable assembly. MOEMS lasers based on FabryPerot external cavities have been demonstrated by NTT [3] These used small mirrors, placed close to an anti-reflection (AR) coated facet of a conventional diode laser and actuated by an electrostatic drive. Because of the length of the cavity, the longitudinal modes were closely spaced, and the spectral properties and tuning characteristics were relatively poor. MOEMS tunable lasers based on vertical cavity semiconductor lasers with much shorter cavities have been developed by several organizations. Bandwidth9 Inc. use an electrostatically deflected cantilever for tuning [4]. An alternative is a deformable membrane mirror, as demonstrated by Stanford University, Stanford, CA, [5]. Finally, Core Tek, Inc., have developed a movable mem-

Manuscript received February 10, 2003; revised June 17, 2003. This work was supported by Marconi plc and the EPSRC. An earlier version of this paper, "Tuning mechanism for a MEMS external cavity laser," was presented at the IEEE/LEOS International Conference on Optical MEMS, Lugano, Switzerland, August 20-23, 2002, paper ThD4 (2002). Subject Editor N. C. Tien.

The authors are with the Optical and Semiconductor Devices Section, Department of Electrical and Electronic Engineering, Imperial College London, London SW7 2BT, U.K. (e-mail: r.syms@ic.ac.uk).

Digital Object Identifier 10.1109/JMEMS.2003.820269 brane, preshaped by stress into a spherical surface, which reduces diffraction loss [6].

Improved external lasers may be constructed using conventional laser diodes, provided the cavity contains a frequency selective element such as a grating. The most common geometries are the Littrow (e.g., [7]) and Littman [8] configurations. The Littrow cavity uses a single pass through a grating, which is rotated to tune the wavelength. The cavity consists of a semiconductor optical amplifier (SOA) with one facet high-reflection (HR) coated and the other AR-coated, an AR-coated lens and a blazed reflection grating. The Littman cavity uses a double pass through a grating, and requires an additional mirror, which is rotated for tuning. Spectral purity is enhanced in this geometry, because the grating is encountered twice per roundtrip.

The main problems are to ensure thermal and mechanical stability, high wavelength selectivity, and wide-range, mode-hop free tuning. Mode hopping occurs because the cavity supports discrete longitudinal modes. To ensure that the same mode is always selected by the grating, the cavity length must alter during tuning. This may be achieved by inducing a phase shift in the active element by carrier injection [9], or translating the grating [10]. The combined effect of translation and rotation can be achieved using a single motion, by rotating the tuning element about a remote point [11], [12].

Miniature external cavity lasers with blazed gratings were developed at British Telecom Research Labs in the early 1980s [13], [14]. The gratings were mounted in Littrow and tuned using piezoelectric elements. However, the only MEMS tunable laser is the Apollo laser developed by Iolon, Inc., which uses a comb electrostatic actuator to rotate the mirror in a Littman cavity [15]. The actuator is fabricated by deep-reactive ion etching (DRIE) [16] of bonded silicon-on-insulator (BSOI). DRIE of BSOI yields devices with a high aspect ratio and out-of-plane stiffness [17] and high quality optical surfaces, such as mirror insertion switches [18]. In earlier work, we have shown that DRIE may also be used to form blazed gratings (albeit with limited resolution) [19].

In this paper, we describe the integration of these gratings with a suspension and drive, to form a low cost-tuning element. The suspension is a compound flexure, introduced in [20]. Initial prototypes had relatively poor performance. Here we present an improved design, which confirm the basic concept. The theory of Littrow cavity tuning is briefly reviewed in Section II. The design of a flexure suspension capable of acting as a pivot is presented in Section III. Fabrication of tuning elements and electromechanical characterization are described in Section IV. Electrical tuning of a Littrow cavity laser is demonstrated in Section V and conclusions are presented in Section VI. 


\section{TUNING OF A LITTROW CAVITY}

We first review the theory of an ideal pivot [10], [11]. In the Littrow geometry, longitudinal resonance is satisfied when $\lambda=2 L_{c} / m$. Here, $m$ is an integer, $\lambda$ is the wavelength, and $L_{c}$ is the optical cavity length (i.e., the sum of the product of the length and refractive index of all intracavity components, including the gain block, collimating lens and air space). Now, $n$ th-order retro-reflection from a blazed grating of period $\Lambda$ at an angle $\theta$ to the oncoming beam occurs at wavelengths when $\lambda=\{2 \Lambda / n\} \sin (\theta)$. To match the resonance with the peak in grating reflectivity, the cavity length should therefore initially be adjusted so that both conditions are satisfied, when

$$
L_{c}=\left\{\frac{m \Lambda}{n}\right\} \sin (\theta) .
$$

To maintain this condition when the grating is rotated by an angle $d \theta$, the cavity length must alter by a length $d L_{c}$, such that $d L_{c} / d \theta=\{m \Lambda / n\} \cos (\theta)=L_{c} / \tan (\theta)$. To first order, this can be achieved by mounting the grating on a radius arm extending tangentially from the grating, choosing the radius $R$ so that

$$
R=\frac{L_{c}}{\sin (\theta)} .
$$

Note that (2) is independent of both the mode number $m$ and the grating order $n$, so the optimum pivot radius is unique. Tuning involves small rotation of the grating. Differentiating (2), we obtain $d \theta=\tan (\theta) d \lambda / \lambda$. For a laser operating at $\lambda \approx$ $1.5 \mu \mathrm{m}$ wavelength, with the grating set at $\theta \approx 45^{\circ}$, a tuning range of (say) $\Delta \lambda=50 \mathrm{~nm}$ requires rotation through an angular range of $\Delta \theta \approx 0.033 \mathrm{rad}$, or $2^{\circ}$. This rotation involves a linear tangential motion $\Delta$ of the grating of $\Delta=R \Delta \theta$. For a radius of (say) $R \approx 2 \mathrm{~mm}$, the linear tangential motion is therefore of the order of 66 microns, well within the capability of MOEMS.

\section{FleXure Mount FOR Synchronous Tuning}

We now consider the design of an appropriate flexure mount, which should ideally have the following attributes: 1) a primary end-displacement that mimics rotation about an optimized pivot, and 2) a secondary linear motion that allows adjustment of the cavity length, without altering the grating orientation. This second motion is required to adjust the cavity to satisfy (1). The mount used in the Iolon Apollo ${ }^{\mathrm{TM}}$ laser has the form of a four-bar linkage, based on rigid bars hinged by flexures [15]. Here, we consider alternative designs based on continuous flexures.

Any point on an elastic structure will have (to first order) a fixed relation between its linear and angular deflections, and hence an instantaneous centre of rotation. For structures based on combinations of parallel beam elements, the centre may be found analytically. For example, the tip of an end-loaded cantilever of length $L$ has an instantaneous centre $L / 3$ from its root, and may therefore satisfy condition 1 ) if the pivot is located there. However, it cannot easily satisfy condition 2). Fig. 1(a) shows a structure that allows separate adjustment of linear and angular displacements. It consists of a cantilever of length $L_{2}$ a)

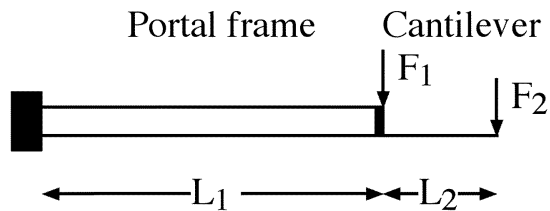

b)

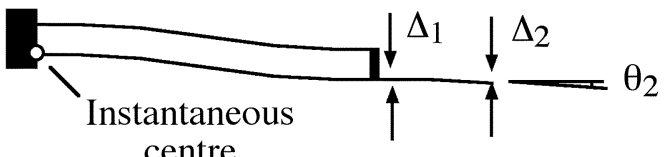

centre

c)

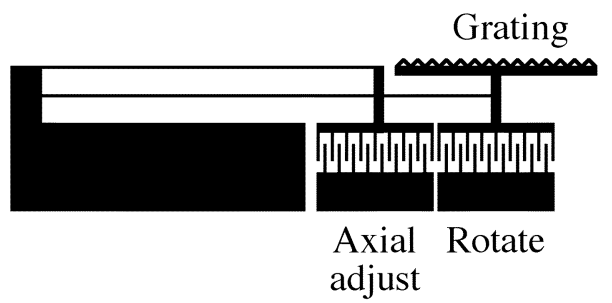

d)

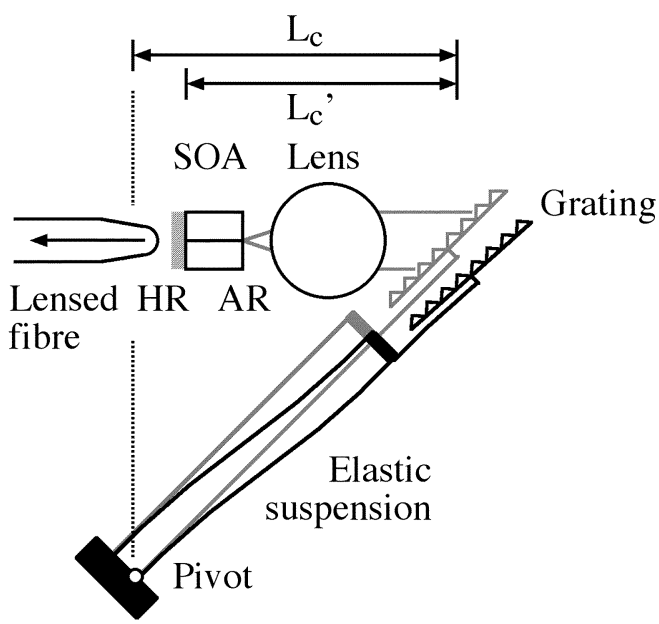

Fig. 1. (a) Undeflected and (b) deflected compound flexure suspension; (c) grating suspension with independent angular and linear comb drive actuators; (d) arrangement of tuning element in a Littrow external cavity laser.

attached to a portal frame of length $L_{1}$. If a point load $F_{2}$ is applied to the cantilever tip, the linear and angular displacements $\Delta$ and $\theta_{2}$ are

$$
\Delta=\Delta_{1}+\Delta_{2}=F_{2}\left\{\frac{1}{k_{1 L}}+\frac{1}{k_{2 L}}\right\} \text { and } \theta_{2}=F_{2}\left\{\frac{1}{k_{2 A}}\right\} .
$$

Here, the linear stiffness $k_{1 L}$ of the portal frame, and the linear and angular stiffnesses $k_{2 L}$ and $k_{2 A}$ of the cantilever are given by

$$
k_{1 L}=\frac{24 E I_{1}}{L_{1}^{3}}, \quad k_{2 L}=\frac{3 E I_{2}}{L_{2}^{3}} \text { and } k_{2 A}=\frac{2 E I_{2}}{L_{2}^{2}}
$$

where $I_{1}$ and $I_{2}$ are the second moments of the portal and cantilever flexures, respectively.

The use of a two-section design allows some choice in the location of the pivot. For example, to locate the pivot at the root of the portal, as in Fig. 1(b), we require

$$
\Delta=\left\{L_{1}+L_{2}\right\} \theta_{2} .
$$


Equation (5) can be satisfied by careful choice of the ratio $\alpha=L_{1} / L_{2}$. In the special case when $I_{1}=I_{2}$ (i.e., for similar beams), $\alpha$ is the solution of the cubic equation:

$$
\alpha^{3}-12 \alpha-4=0 .
$$

Equation (6) may be solved numerically, to give $\alpha=3.62$. This ratio may be determined to high accuracy in a vertically etched MEMS structure by assuming equal widths for the portal and cantilever beams, and setting $L_{1}=3.62 L_{2}$ at mask-making.

If, alternatively, the point load $F_{1}$ is applied to the portal frame, the displacements are

$$
\Delta=F_{1}\left\{\frac{1}{k_{1 L}}\right\} \text { and } \theta_{2}=0 .
$$

In this case, the cantilever tip will displace linearly without rotating.

A complete grating tuning element may be constructed as shown in Fig. 1(c). The grating is mounted on the cantilever tip, and the point loads $F_{1}$ and $F_{2}$ are applied by (for example) two comb electrostatic actuators. One is used to rotate the grating about the pivot point, while the other provides a linear translation to adjust the cavity length. The composite structure may be used in a Littrow cavity laser as shown in Fig. 1(d). Here, the tuning element provides a pivot for a cavity of optical length $L_{c}$, which is greater than $L_{c}^{\prime}$, the sum of the physical lengths of the intracavity components. For short cavities, the difference between $L_{c}$ and $L_{c}^{\prime}$ may be large.

\section{REALISATION OF LASER TUNING ELEMENTS}

Complete tuning elements consisting of blazed gratings, elastic elements and comb drives were fabricated by deep reactive ion etching and undercut of bonded silicon-on-insulator material. The BSOI consisted of $4^{\prime \prime}$ dia (100) orientated Si, with a bonded layer thickness of $75 \mu \mathrm{m}$ and an oxide interlayer thickness of $2 \mu \mathrm{m}$.

The DRIE was carried out in an inductively coupled plasma etcher, using a standard cyclic etch-passivate process and a hard mask [16]. After etching, the mask was stripped, and the oxide interlayer was removed from beneath the suspended parts by wet etching in buffered HF. The structures were then freeze-dried in a water/methanol mixture and metallized with $200 \AA$ of $\mathrm{Cr}$ and $300 \AA$ of Au to improve reflectivity and allow electrical contact. Isolation between the different sections was ensured by the long undercut etch, which prevented metal tracking over the supporting pads of oxide.

In the experimental devices, the portal length was $L_{1}=$ $1.81 \mathrm{~mm}$ and the cantilever length was $L_{2}=0.5 \mathrm{~mm}$, so that $R=2.31 \mathrm{~mm}$. The width of all flexures was $6 \mu \mathrm{m}$, and the width of the strut linking the portal beams was $18 \mu \mathrm{m}$. The beams in the portal frame were spaced apart by a distance of $50 \mu \mathrm{m}$. The comb drives contained 21 movable and 20 fixed fingers, each with a width and separation of $6 \mu \mathrm{m}$ and a length of $110 \mu \mathrm{m}$. The static overlap of the fingers was small $(10 \mu \mathrm{m})$, for reasons given later.

The blaze angle was chosen to be $45^{\circ}$, to simplify mask layout. Gratings were fabricated with periods of $1.5 \sqrt{ } 2,3 \sqrt{ } 2$,
$4.5 \sqrt{ } 2,6 \sqrt{ } 2,7.5 \sqrt{ } 2$, and $9 \sqrt{ } 2 \mu \mathrm{m}$, corresponding to second, fourth, sixth, eighth, tenth, and twelfth order at $\lambda=1.5 \mu \mathrm{m}$. The overall grating width was approximately $500 \mu \mathrm{m}$. The grating was supported on a beam of length $600 \mu \mathrm{m}$, to provide an additional $50 \mu \mathrm{m}$ long plane reflector on either end of the grating. These mirrors were used to measure the motion.

Using bare silicon wafers, it was found that the majority of the structure could be etched to a depth of $150 \mu \mathrm{m}$, before a hard mask of $3 \mu \mathrm{m}$ thick photoresist was completely removed by the plasma. However, there was considerable variation in the feature height, and the narrow regions between the comb electrodes were considerably under-etched compared to the exposed front surface of the blazed grating.

Similar effects occurred on BSOI wafers, except that a resist hard mask was eroded very rapidly during the stop-on-oxide step, which requires the use of low frequency plasma. The resist mask was therefore reinforced by the addition of $2000 \AA \mathrm{Cr}$ metal beneath, and etching was continued for a calibrated time (estimated from experiments on bare $\mathrm{Si}$ wafers) after the majority of the wafer had been etched down to the oxide.

Using this strategy, devices could be formed in $75 \mu \mathrm{m}$ thick bonded layers across the whole wafer. Gratings of eighthtwelfth order were transferred relatively well in the process, albeit with some rounding of the tooth profile. However, loworder gratings were not transferred to any useable depth. Fig. 2(a) shows a completed device, Fig. 2(b) an enlarged view of the electrodes and Fig. 2(c) the surface of a twelfth-order grating.

Completed elements were characterized interferometrically as shown in Fig. 3(a). Broad-band light centered on $\lambda=1535 \mathrm{~nm}$ from an Agilent 83438A erbium ASE source was coupled into Port 1 of a 3 -dB single-mode fiber coupler. Port 4 was placed near the device so that a Fabry-Pérot cavity was set up between the cleaved fiber and the vertically etched surface. The length of this cavity was $\approx 350 \mu \mathrm{m}$. Reflected light was measured from Port 2, using an Agilent 86140B optical spectrum analyzer (OSA). Port 3 was placed in index matching oil. The spectral variation of reflectivity contained a periodic modulation, from which the displacement of different points could be found.

Measurements of displacement $\Delta_{A}$ and $\Delta_{B}$ were made at the points $\mathrm{A}$ and $\mathrm{B}$ (the left- and right-hand of the grating) under the influence of the two comb drives, so that the grating motion could be deduced. First, the effect of the axial drive was investigated. The two displacements were found to vary linearly with the square of the applied voltage, as expected for a comb-drive actuator. They were also highly similar, implying that the axial drive can induce translation of the grating, without rotation.

The effect of the angular drive was then investigated. The displacements $\Delta_{A}$ and $\Delta_{B}$ again varied quasilinearly with voltage squared as shown in Fig. 3(b), but the slopes of the characteristics were different, suggesting that the grating was now rotating. The data were interpolated, to give values of displacement for a given voltage, and the angle of rotation was found as $\theta_{2}=\left\{\Delta_{B}-\Delta_{A}\right\} / W$, where $W$ is separation between $\mathrm{A}$ and $\mathrm{B}$. The variation of angle with voltage squared is shown in Fig. 3(c). At low voltages, the variation is linear. However, at higher voltages, the slope increases. 


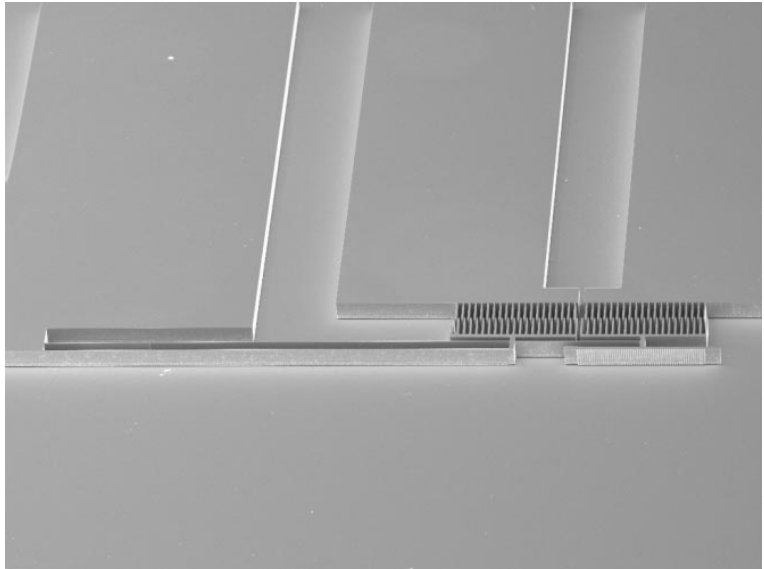

(a)

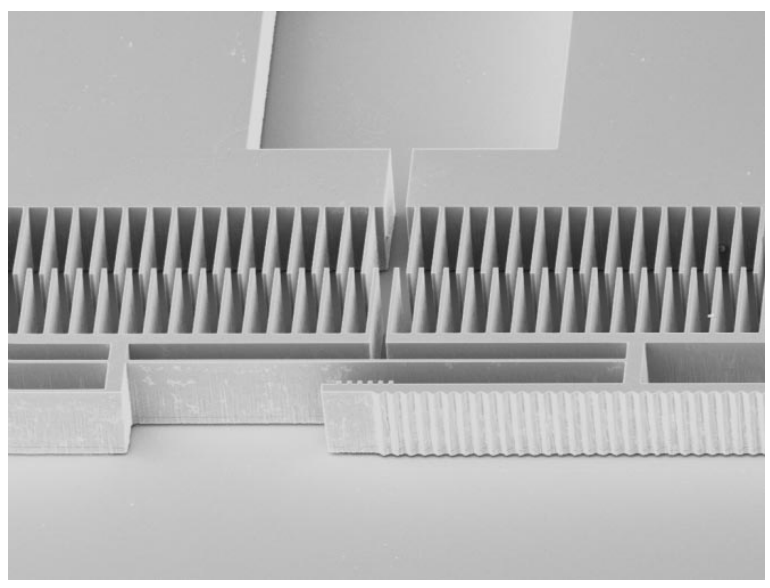

(b)

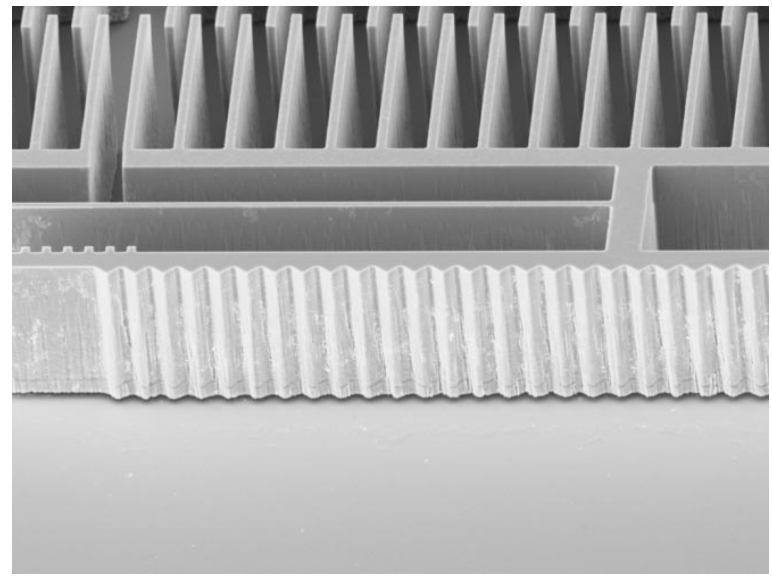

(c)

Fig. 2. (a) Overall view of fabricated device in $75 \mu \mathrm{m}$ thick BSOI; (b) detail of drive electrodes; and (c) detail of twelfth-order grating.

The instantaneous pivot radius was found as $R=$ $\left\{\Delta_{B}+\Delta_{A}\right\} / 2 \theta_{2}$. This variation is shown in Fig. 3(c), plotted as $R / R_{\text {ideal }}$. The calculation is strongly affected by errors in the data, and measurements must be made very carefully at low angles. $R$ is not constant, but falls slightly with angle, from an initial value that corresponds very well with the design. These results suggest that the instantaneous pivot must move slightly toward the grating as it rotates, varying by around $25 \%$ over the angular range shown.
The explanation is an additional torque $T_{2}$, acting at the cantilever tip as shown in Fig. 4(a) and arising from unbalanced electrostatic forces. Two sets of forces act in a comb actuator. The first draw the two electrodes together, and are used for conventional actuation. The second act perpendicularly between each finger and its neighbors. Usually the lateral forces balance, to leave only the normal forces. However, rotation of the cantilever tip skews the movable electrode, so that the separations between movable fingers and their fixed neighbors on either side are no longer equal. Lateral forces then become unbalanced and give rise to the torque $T_{2}$, which increases the rotation further.

The severity of this effect depends on the distance $D$ from the radius arm to the tip of the moving electrodes. In the previous design [20], $D$ was relatively large (300 $\mu \mathrm{m})$, and highly nonideal behavior was observed. Because the angular variation of the torque $T_{2}$ is nonlinear, it will eventually overcome the suspension stiffness and snap the fingers together. Fig. 4(b) shows an earlier device that reached snap-down after the grating rotated through $1.3^{\circ}$. To first order, all moving fingers have contacted the fixed fingers simultaneously [20]. In the design here, $D$ is reduced to $150 \mu \mathrm{m}$, by minimizing the lengths of the fingers and supporting struts. The onset of the electrostatic instability is therefore postponed, and good performance is obtained over a larger angular range.

Measurements were also made with voltages applied to both actuators simultaneously. For example, a fixed voltage was applied to the axial drive, and a variable voltage to the angular drive. A similar angular motion was obtained (at least for small displacements) as the angular drive voltage varied, suggesting independent operation of the actuators. However, at large deflections the portal frame will follow a curved locus, which will result in a second-order lateral deflection of the comb electrodes. This unwanted deflection may in turn give rise to coupling between the angular and axial motions. Clearly, this aspect merits further attention.

\section{TunABle LitTrow External CAVITy LASER}

Littrow external cavity lasers were constructed using twelfthorder gratings, previously described in [19]. Spectral properties were measured using an optical spectrum analyzer and a 1550-nm edge emitting LED source as in Fig. 5(a). The incident beam was collimated by a 0.5 -mm diameter, 0.23 -pitch gradient index rod lens (GRINTECH GmbH, Germany), and the reflected beam was separated by a 3 -dB fiber coupler. Fig. 5(b) shows the response of a $150-\mu \mathrm{m}$-deep grating. Here, the reflectivity is compared with a plane, Au-metallized Si surface. The peak reflectivity is $5.5 \mathrm{~dB}$ lower than the mirror, and the half-power bandwidth is $\approx 20 \mathrm{~nm}$.

External cavity lasers were constructed using twelfth-order gratings mounted on MEMS tuning elements. The gain block was a stripe guide InGaAsP device from OptoSpeed (LCSH1550-TAP). Light from the AR coated facet was collimated by a 0.5-mm-diameter ball lens (Edmund Optics). Emission from the HR coated facet was coupled into a lensed single-mode fiber (Seikho Gihken), which was then connected to the OSA. The lensed tip radius was $9 \mu \mathrm{m}$, and the tip was AR coated for $1550 \mathrm{~nm}$ wavelength. 


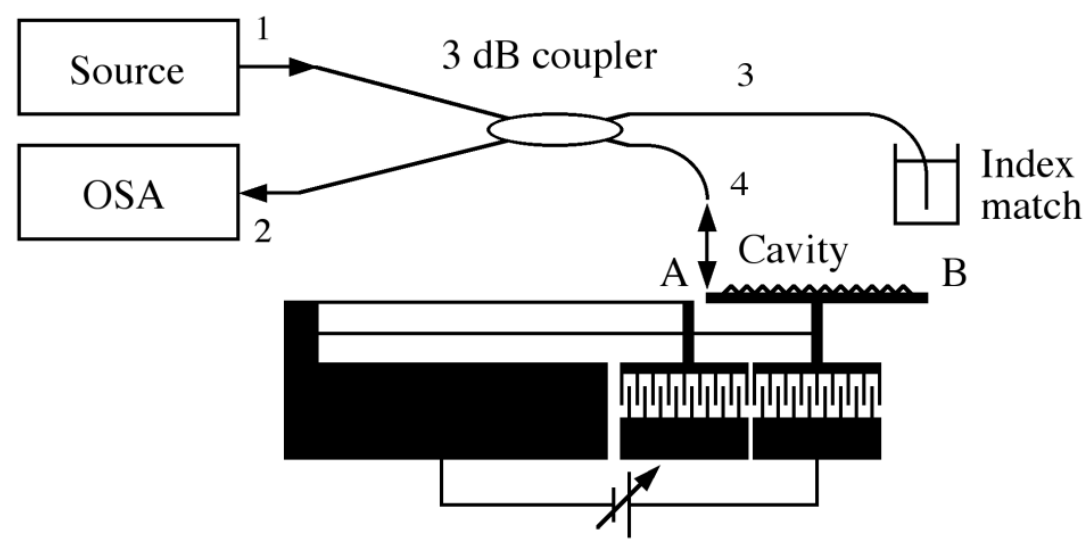

(a)

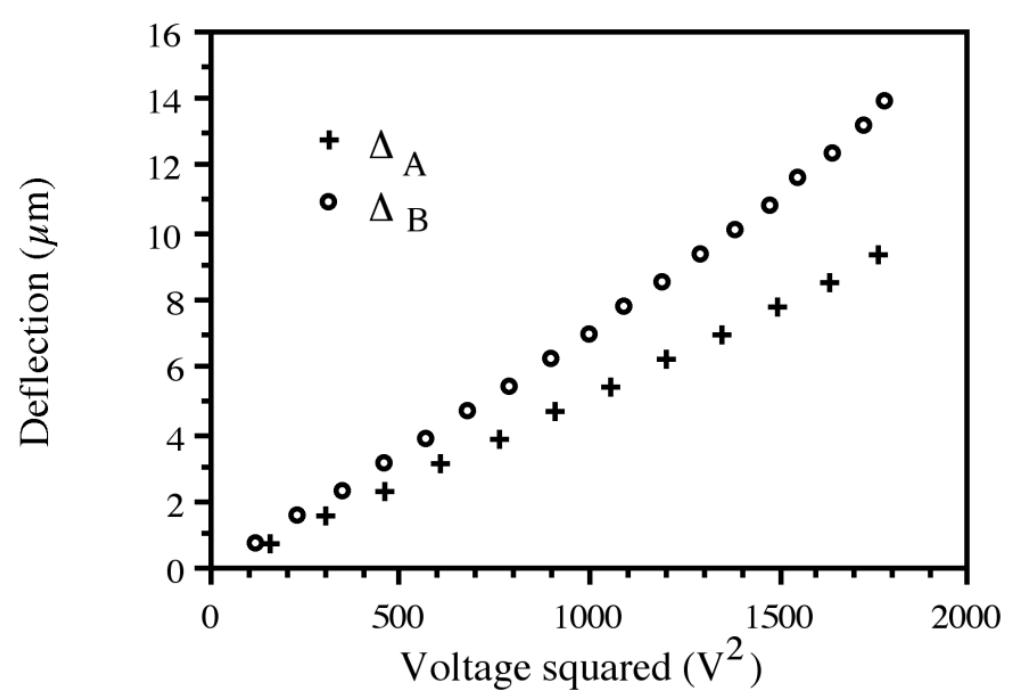

(b)

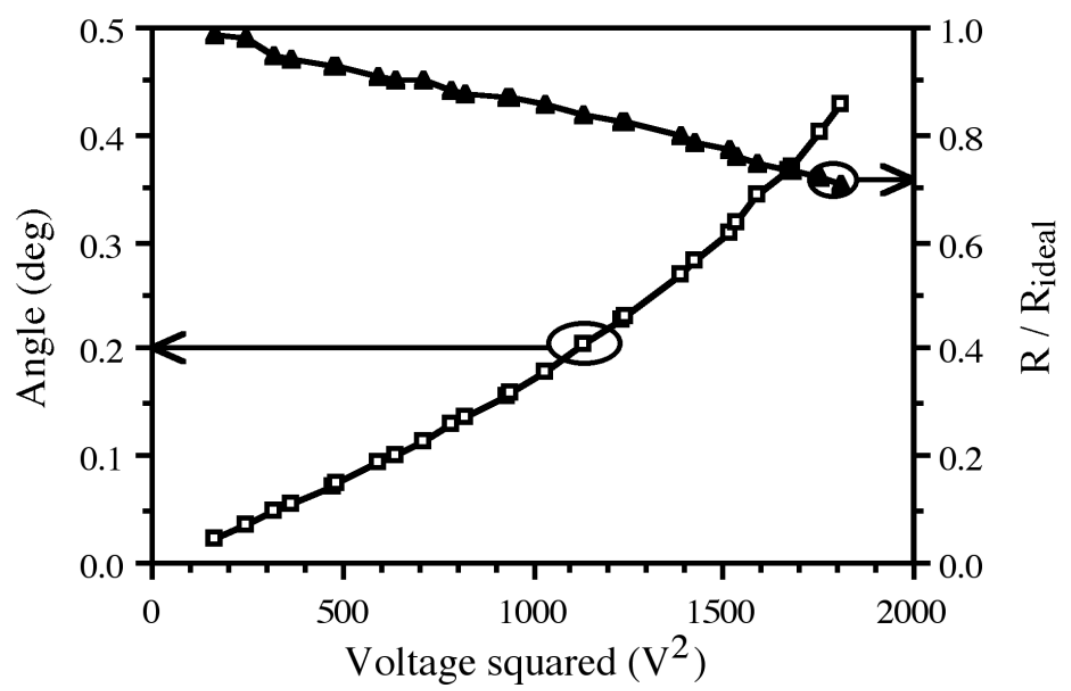

(c)

Fig. 3. (a) Arrangement for interferometric displacement measurement. (b) Variation of displacements $\Delta_{A}$ and $\Delta_{B}$ with the square of the voltage applied to the angular tuning actuator. (c) Similar variation of grating angle $\theta_{2}$ and instantaneous pivot radius $R$.

Lasers were constructed with the grating at $45^{\circ}$ incidence. At this angle, the appropriate effective cavity length is $L_{c}=$ $R / \sqrt{ } 2 \approx 1.63 \mathrm{~mm}$. For the particular SOA and lens used, the required air space is then extremely small $(\approx 100 \mu \mathrm{m})$. Since the smallest practical space was found to be $\approx 1.3 \mathrm{~mm}$, the pivot could not be located at the ideal point. The tuning element, lens, 


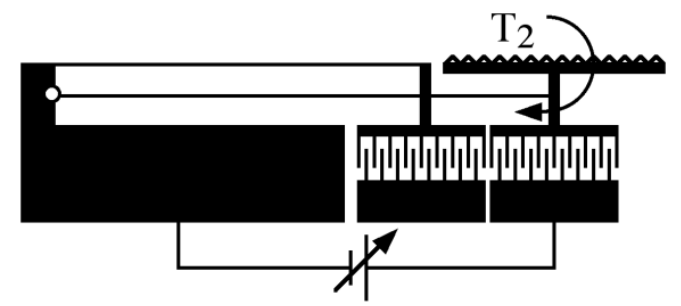

$\overline{1}$

(a)

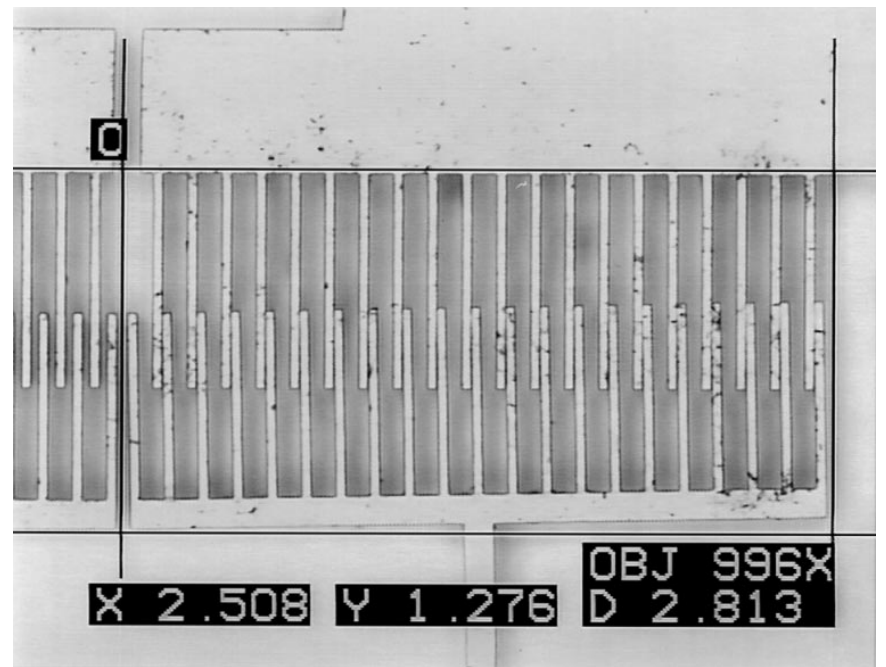

(b)

Fig. 4. (a) Definition of additional torque $T_{2}$. (b) Microscope view of the overdriven actuator.

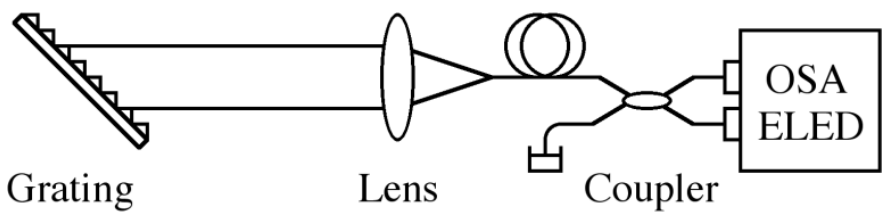

(a)

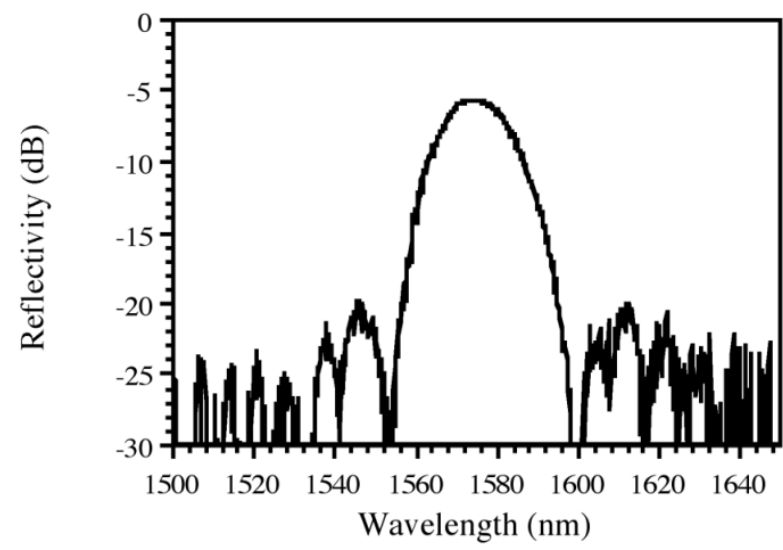

(b)

Fig. 5. (a) Arrangement for measurement of grating performance. (b) Bandpass characteristic of twelfth-order grating fabricated by deep etching (from [19]).

SOA and lensed fiber were all held on separate manipulators to align the cavity. The MEMS part was mounted on a two-axis rotation stage and a three-axis translation stage. Fig. 6(a) shows

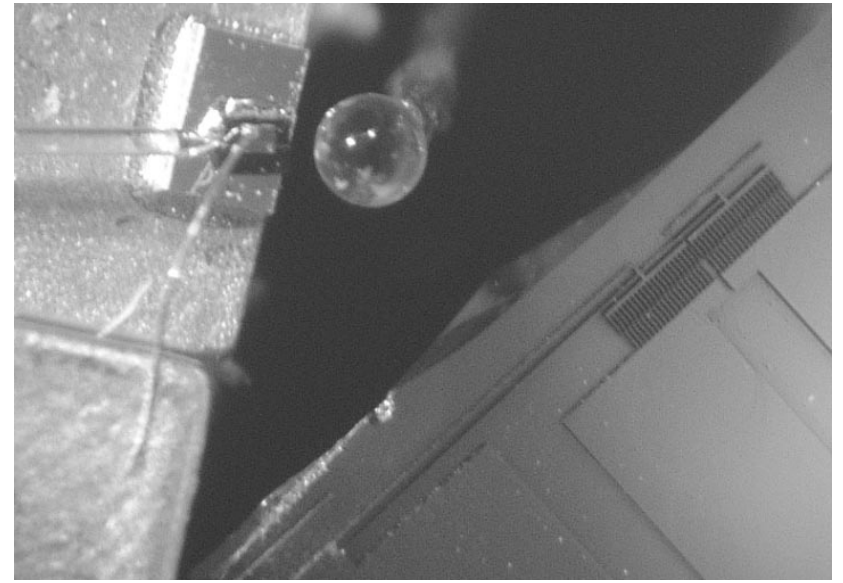

(a)

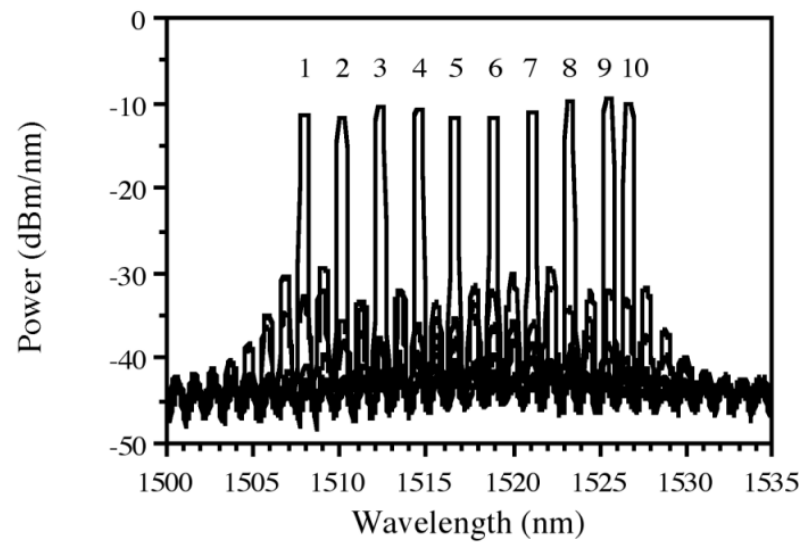

(b)

Fig. 6. (a) Layout of Littrow external cavity laser. (b) Emission spectra obtained with 10 different voltages applied to the angular tuning electrode. The corresponding actuator drive voltages may be found from Fig. 7(a).

a laser under test; the tuning element, SOA, lens and fiber may all be seen. The SOA is skewed to prevent multiple reflections.

Although Fig. 5(b) shows that the gratings have limited spectral selectivity, they can still yield reasonable performance from an external cavity laser, as demonstrated by the laser emission spectra shown in Fig. 6(b). Here, low drive currents (35 mA) were used, to avoid damaging the SOA. The emission is clearly single mode, with a side-mode suppression ratio (SMSR) of $>20 \mathrm{~dB}$. The peak fiber-coupled power is $-10 \mathrm{dBm}(100 \mu \mathrm{W})$. Output powers up to $500 \mu \mathrm{W}$ were obtained in earlier experiments [20], after which the emission became multimoded.

Further increases in single mode power and SMSR require a more selective filter. This would be obtained using a lower order grating (which would require improved pattern transfer) and an increased illuminated area (which requires the use of a thicker bonded silicon layer). In fact, an output power of $\approx 1 \mathrm{~mW}$ and a SMSR of $\approx 30 \mathrm{~dB}$ have already been achieved using fixed gratings etched to a structural depth of $150 \mu \mathrm{m}$ [19].

The different spectra are obtained with increasing voltage applied to the angular tuning actuator. For low voltages, the emission shifts steadily to longer wavelength, over a spectral range of $\Delta \lambda=20 \mathrm{~nm}$ from an initial value of $\lambda=1508 \mathrm{~nm}$, using a maximum voltage of ca $50 \mathrm{~V}$. The use of an incorrect cavity length did give rise to mode hopping during tuning. Additional 


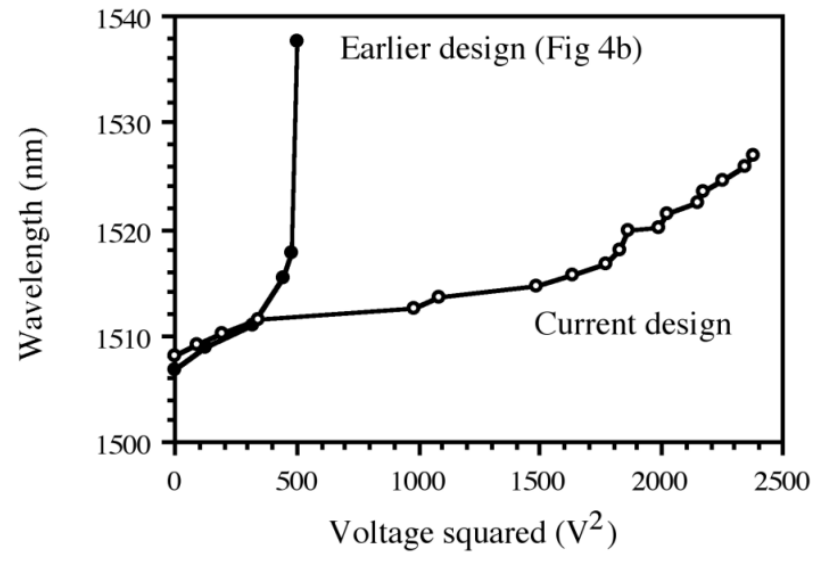

(a)

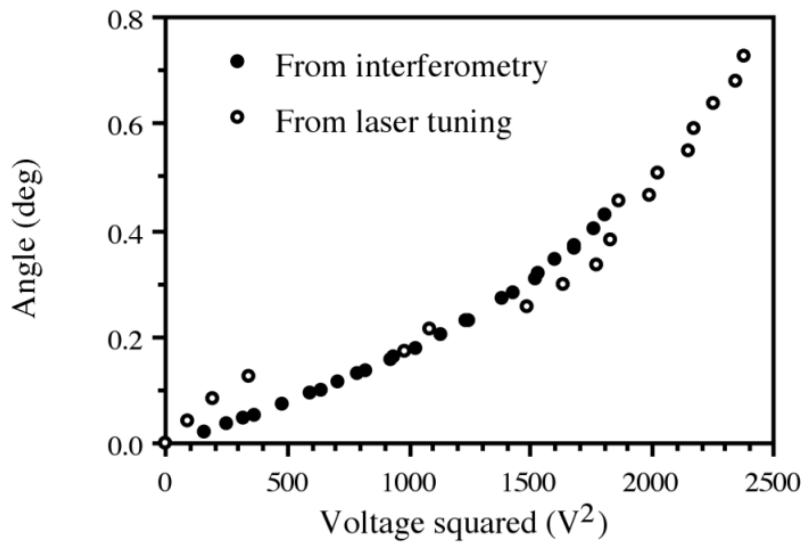

(b)

Fig. 7. (a) Variation of emission wavelength with voltage squared. (b) Comparison of angular responses obtained by interferometric measurement and laser tuning.

mode hopping was observed even under steady state conditions. The assumed cause is the limited stability of the stacked micromanipulator stages, although there may be additional instability in the MEMS device itself.

Fig. 7(a) shows the variation of emission wavelength with the square of the voltage applied to the angular tuning actuator. The tuning characteristic is quasilinear for low voltages. However, its slope gradually increases with voltage. This behavior is clearly related to the electrostatic instability described in Section IV. Superimposed on Fig. 7(a) are similar results for the earlier design that failed catastrophically in Fig. 4(b) [20]. The increase in tuning range using the improved design is marked. Fig. 7(b) compares the angular variation with voltage obtained from interferometric and laser tuning measurements. There is clearly good correlation between the two, with the differences being primarily ascribed to mode hopping during tuning.

These preliminary experiments show that an electrostatically actuated grating may be integrated using MOEMS techniques, and confirm the basic device concept.

\section{CONCLUSION}

A MOEMS tuning element for an external cavity laser has been developed by combining a blazed grating, a flexure suspension, and comb-drive electrostatic actuators using a simple fabri- cation process based on DRIE of BSOI material. The elastic suspension is designed to simulate the operation of an ideal pivot, and is based on a cantilever coupled to a portal frame.

Electromechanical performance has been characterized using an interferometer, and these experiments have shown that the tuning element may be translated and rotated independently. The actuator is well behaved for small angular deflections, but then departs from the required motion. This behavior is attributed to an electrostatic instability caused by lateral deflection of the electrode fingers. Work is in progress to develop an actuator capable of applying the point load required for correct operation.

The tuning element has been used in a Littrow external cavity laser based on a commercial SOA. Vertically etched gratings have been sufficiently reflective that $100 \mu \mathrm{W}$ output power has been obtained into a single mode optical fiber. Although only high order gratings have so far been fabricated, their spectral selectivity is sufficient to obtain single mode operation, with a side-mode suppression ratio of $-20 \mathrm{~dB}$. Improvements in power and SMSR would follow from improvements in grating quality. Controllable tuning over a range of $20 \mathrm{~nm}$ has been demonstrated, and work is continuing to improve the linearity of tuning and the tuning range.

\section{ACKNOWLEDGMENT}

The assistance of Dr. J. Stagg with deep etching is also gratefully acknowledged.

\section{REFERENCES}

[1] V. Jayaraman, Z. M. Chuang, and L. A. Coldren, "Theory, design and performance of extended tuning range semiconductor lasers with sampled gratings," IEEE J. Quantum Electron., vol. QE-29, pp. 1824-1834, 1993.

[2] G. Morthier and G. Sarlet, "Widely tunable laser diodes and their control," in Proc. 25th Eur. Conf. Optical Communication (ECOC), vol. II, Nice, France, Sept. 26-30, 1999, pp. 118-119.

[3] Y. Uenishi, K. Honma, and S. Nagaoka, "Tunable laser diode using a nickel micromachined external mirror," Electron. Lett., vol. 32, pp. 1207-1208, 1996.

[4] M. Y. Li, G. S. Li, and C. J. Chang-Hasnain, "Top-emitting micromechanical VCSEL with a $31.6 \mathrm{~nm}$ tuning range," IEEE Photon. Technol. Lett., vol. 10, pp. 18-20, 1998.

[5] F. Sugihwo, M. C. Larson, and J. S. Harris, "Micromachined widely tunable vertical cavity laser diodes," J. Microelectromech. Syst., vol. 7, pp. 48-55, 1998.

[6] D. Vakhshoori, P. Tayebati, C.-C. Lu, M. Azimi, P. Wang, J.-H. Zhou, and E. Canoglu, " $2 \mathrm{~mW} \mathrm{CW}$ singlemode operation of a tunable $1550 \mathrm{~nm}$ vertical cavity surface emitting laser with $50 \mathrm{~nm}$ tuning range," Electron. Lett., vol. 35, pp. 900-901, 1999.

[7] H. Lotem, "Littrow-mounted diffraction grating cavity," Appl. Opt., vol. 33, pp. 930-934, 1994.

[8] M. G. Littman and H. J. Metcalf, "Spectrally narrow pulsed dye laser without a beam expander," Appl. Opt., vol. 17, pp. 2224-2227, 1978.

[9] C. Petridis, I. D. Lindsay, D. J. M. Stothard, and M. Ebrahimzadeh, "Mode-hop-free tuning over $80 \mathrm{GHz}$ of an extended cavity diode laser without antireflection coating," Rev. Sci. Inst., vol. 72, pp. 3811-3815, 2001.

[10] F. Favre, L. D. Guen, J. C. Simon, and B. Landousies, "External cavity semiconductor laser with $15 \mathrm{~nm}$ continuous tuning range," Electron. Lett., vol. 22, pp. 795-796, 1986.

[11] F. Favre and D. Le Guen, " $82 \mathrm{~nm}$ of continuous tunability for an external cavity semiconductor laser," Electron. Lett., vol. 27, pp. 183-184, 1991.

[12] W. R. Trutna and L. F. Stokes, "Continuously tuned external cavity semiconductor laser," J. Lightwave Technol., vol. 11, pp. 1279-1286, 1993. 
[13] J. Mellis, S. A. Al-Chalabi, K. H. Cameron, R. Wyatt, J. C. Regnault, W. J. Devlin, and M. C. Brain, "Miniature packaged external-cavity semiconductor laser with $50 \mathrm{GHz}$ continuous electrical tuning range," Electron. Lett., vol. 24, pp. 988-989, 1988.

[14] M. Bagley, R. Wyatt, D. J. Elton, H. J. Wickes, P. C. Spurdens, C. P. Seltzer, D. M. Cooper, and W. J. Devlin, "242 nm continuous tuning from a GRIN-SC-MQW-BH InGaAsP laser in an extended cavity," Electron. Lett., vol. 26, pp. 267-269, 1990.

[15] J. D. Berger, Y. Zhang, J. D. Grade, H. Lee, S. Hrinya, H. Jerman, A. Fennema, A. Tselikov, and D. Anthon, "Widely tunable external cavity diode laser using a MEMS electrostatic rotary actuator," in Proc. 27th European Conf. on Optical Communications, vol. 2, Amsterdam, Sep.-Oct. 30-4, 2001, pp. 198-199.

[16] J. K. Bhardwaj and H. Ashraf, "Advanced silicon etching using high density plasmas," Proc. SPIE, vol. 2639, pp. 224-233, 1995

[17] E. H. Klaassen, K. Petersen, J. M. Noworolski, J. Logan, N. I. Maluf, J. Brown, C. Storment, W. McCulley, and T. A. Kovacs, "Silicon fusion bonding and deep reactive ion etching: A new technology for microstructures," Sens. Actuators, vol. A52, pp. 132-139, 1996.

[18] C. Marxer, M.-A. Gretillat, N. F. de Rooij, R. Battig, O. Anthamatten, B. Valk, and B. Vogel, "Vertical mirrors fabricated by reactive ion etching for optical fiber switching applications," in Proc. 10th Workshop on MEMS, Nagoya, Japan, 1997, pp. 49-45.

[19] A. Lohmann and R. R. A. Syms, "External cavity laser with a verticallyetched silicon blazed grating," IEEE Photon. Technol. Lett., vol. 15, pp. 120-122, 2003.

[20] R. R. A. Syms and A. Lohmann, "Tuning mechanism for a MEMS external cavity laser," presented at the IEEE/LEOS Int. Conf. on Optical MEMS and their Applications, Lugano, Switzerland, Aug. 20-23, 2002, paper ThD4.

[21] W. C. Young, Roark's Formulas for Stress and Strain, 6th ed. New York: McGraw Hill International, 1989.

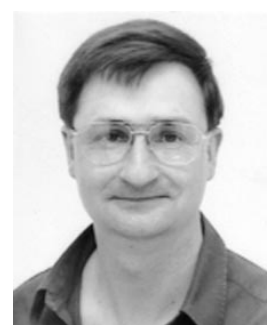

Richard R. A. Syms (M'98-SM'03) was born in Norfolk, VA, in 1958. He received the B.A. degree in Engineering Science in 1979 and the D.Phil. degree (on volume holographic optical elements) in 1982, both from Worcester College, Oxford, U.K.

$\mathrm{He}$ has been Head of the Optical and Semiconductor Devices Group in the Department of Electrical and Electronic Engineering, Imperial College London, U.K., since 1992 and Professor of Microsystems Technology since 1996. He has published approximately 90 papers and two books on holography, integrated optics, and microengineering. His current interests are silica-on-silicon integrated optics and silicon-based MEMS. Most recently, he has been developing microengineered mass spectrometers, optical MEMS devices, and 3-D self-assembling microstructures.

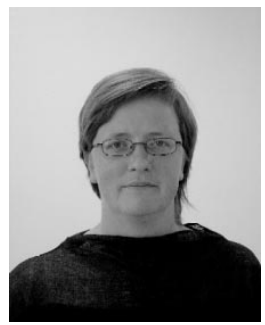

Anke Lohmann was born in 1971 in Halle, Germany. She received the diploma in electronics from the Technical University Dresden, Dresden, Germany, in 1997. Currently, she is working toward the Ph.D. degree on a MOEMS hybrid tunable laser at Imperial College London, U.K.

She has been a member of the Optical and Semiconductor Devices Group in the Department of Electrical and Electronics Engineering, Imperial College London since 2000, where she has been involved with various MEMS projects. Prior to this, she worked at the Institute of Ophthalmology at UCL, London, U.K., on optical coherence tomography and image processing. 